\title{
Risk factors for complications after primary repair of Achilles tendon ruptures
}

\author{
Christian A. Pean ${ }^{\mathrm{a}, \mathrm{b}}$, Anthony Christiano ${ }^{\mathrm{a}, \mathrm{b}}$, William J. Rubenstein ${ }^{\mathrm{a}, \mathrm{b}}$, Sanjit R. Konda ${ }^{\mathrm{a}, \mathrm{b}}$, \\ Kenneth A. Egol ${ }^{\mathrm{a}, \mathrm{b}, *}$
}

${ }^{\text {a }}$ NYU Langone Orthopedic Hospital, Department of Orthopedic Surgery, New York, N.Y., 10003, USA

${ }^{\mathrm{b}}$ Jamaica Hospital Medical Center, Queens, N.Y., USA

\section{A R T I C L E I N F O}

\section{Keywords:}

Achilles tendon rupture

Diabetes

Outcome studies

Tendon disorders

\begin{abstract}
A B S T R A C T
Purpose: To identify patient characteristics associated with adverse events in Achilles tendon rupture (ATR) surgical repair cases.

Methods: A high risk (HR) cohort group of ATR patients were compared to healthy controls in the ACSNSQIP database with multivariate regression analysis.

Results: Overall, $2 \%(\mathrm{n}=23)$ of the group sustained an AE postoperatively, most commonly superficial SSI $(0.9 \%, n=10)$. Multivariate analysis did not reveal any patient characteristics to be significantly associated with the occurrence of an AE or superficial SSI.

Conclusions: Obesity, diabetes and a history of smoking did not predispose patients to significantly more AEs in the 30 day postoperative period following ATR repair in this study.
\end{abstract}

\section{Introduction}

Achilles tendon rupture (ATR) is a fairly common injury that results in substantial morbidity with functional deficits persisting more than two years after the initial injury regardless of treatment. ${ }^{20}$ The majority of Achilles tendon ruptures result from sports and exercise related activity. ${ }^{14,16}$ These injuries occur more in males between the ages of 30 and 50 , with an overall incidence of $6-37$ per 100,000 people. ${ }^{12,17,23}$ There has been an increased incidence in recent years among both men and women ${ }^{13}$ with a peak in the spring that may be due to the increased frequency of adults playing recreational sports. ${ }^{21}$

Broadly speaking, ATR can be treated either surgically or non-surgically. Though surgical treatment has been trending downward, ${ }^{13}$ there is currently no consensus among orthopaedic surgeons as to the optimal management of ATRs. A number of recent studies have compared outcomes and complications between surgical and non-surgical treatment of ATR injuries. The results of these studies have shown lower re-rupture rates in surgically repaired ATR with a higher rate of other complications including infection and nerve injury. ${ }^{1,22,24}$ Given these outcomes, concerns regarding adverse events in the postoperative period may influence the decision to pursue operative management in certain patients. It is important for surgeons to be able to identify those patients who are at a higher risk for experiencing adverse events and tailor treatment accordingly.
The American College of Surgeons National Surgical Quality Improvement Program (ACS-NSQIP) is a multi-institutional outcomes database gaining increasing use in the field of orthopaedics to help analyze the various factors that contribute to adverse surgical outcomes. ${ }^{6,19}$ It contains pre-operative, intra-operative, and 30-day follow up data that is prospectively collected using a standardized methodology resulting in a high quality and robust database. To our knowledge, no one has utilized the NSQIP database to analyze the overall incidence of adverse events and associated risk factors following Achilles tendon surgery. The purpose of this study was to (1) identify patient characteristics associated with the occurrence of adverse events in Achilles tendon rupture and (2) compare outcomes in the perioperative period between patients with multiple risk factors and healthy patients.

\section{Materials and methods}

The ACS-NSQIP database was queried for all primary ATR procedures defined as Current Procedural Terminology (CPT) codes 27650 (1085 cases) and 27652 (79 cases) performed in the years 2005-2012. Percutaneous ATR was not included in this analysis in order to have a more uniform cohort. Patients with missing demographic or outcomes information were excluded from analysis.

Adverse events were defined as the occurrence of any of the

\footnotetext{
* Corresponding author at: 301 East 17th Street New York, NY 10003, USA

E-mail address: kenneth.egol@nyumc.org (K.A. Egol).
} 
following: Death, coma $>24 \mathrm{~h}$, ventilator $>48 \mathrm{~h}$, unplanned intubation, stroke/cerebrovascular accident, deep venous thrombosis, pulmonary embolism, cardiac arrest, myocardial infarction, acute renal failure, sepsis, septic shock, return to operating room, wound dehiscence, deep SSI, organ/space infection, graft prosthesis failure, peripheral nerve injury, superficial surgical site infection (SSI), urinary tract infection, pneumonia, or progressive renal insufficiency. Superficial surgical site infection is defined in the NSQIP database as an open wound, cellulitis (erythema, tenderness and swelling), or wound infection was noted postoperatively at the surgical site. A high risk (HR) cohort group was created by compiling patients who were either obese (Body Mass Index [BMI] > 30), had a history of diabetes, or a history of smoking. These patients were compared for rates of adverse events to healthy control patients who had none of these risk factors using Pearson's Chi square test for significant differences (p-value $<0.05$ ). A multivariate logistic regression analysis of the overall cohort including obesity, history of smoking, diabetes, consumption of greater than two drinks of alcohol/day, age, gender, hypertension, and history of chronic obstructive pulmonary disease was completed to assess for independent predictors of adverse events. The model explained $3.4 \%$ of variance in adverse events and correctly classified $98.0 \%$ of cases. A subgroup analysis was completed to compare primary ATR repair with and without graft to ensure the patient groups could be combined and were comparable with regard to outcomes.

\section{Results}

In total, one thousand one hundred and sixty-four patients met inclusion criteria for the study. A subgroup analysis of the two CPT procedures included (27650 and 27652) revealed ATR repair patients with graft differed only in operative time compared to ATR repair patients without graft $(58.34 \pm 26.9 \mathrm{~min}$, vs. $93.25 \pm 40.41 \mathrm{~min}$, $\mathrm{p}<0.001$ ). There was no statistically significant difference in the two procedure groups with regard to adverse events with only one patient in the ATR repair with graft group having a complication compared to 22 in the ATR repair without graft group.

From the overall cohort, six hundred and fifteen met criteria for the HR cohort (53\%). Complete patient demographics are listed in Table 1. Overall, $2 \%(n=23)$ of the group sustained an adverse event during the 30 day postoperative period, the most common being superficial SSI $(0.9 \%, \mathrm{n}=10)$. Frequency of specific complications in the overall cohort and the HR group are included in Table 2. There was no significant difference in the HR cohort versus controls in rates of adverse events

Table 1

Patient characteristics for overall Achilles tendon rupture repair cohort.

\begin{tabular}{lll}
\hline & Overall $(\mathrm{N}=1164)$ & High Risk $(\mathrm{N}=615)$ \\
\hline Demographic & & \\
Age, years & $44.82 \pm 13.32$ & $48.19 \pm 14.12$ \\
$\quad$ Female sex (\%) & $25.6 \%$ & $29.4 \%$ \\
Preoperative Comorbidities & & \\
$\quad$ BMI, kg/m & & $33.79 \pm 6.70$ \\
Functional status (dependent) & 0.1 & 0.2 \\
$\quad$ (\%) & 12.2 & \\
Smoker within 1 year (\%) & 0.9 & 76.9 \\
Alcohol use (\%) & 1.1 & 1.1 \\
Steroid use (\%) & 0.1 & 1.3 \\
$\quad$ Weight loss (\%) & 5.0 & 0.2 \\
$\quad$ Diabetes (\%) & 22.5 & 10.4 \\
Hypertension (\%) & 0.8 & 34.0 \\
COPD (\%) & 1.0 & 1.1 \\
$\quad$ Open wound/wound infection & & \\
$\quad$ (\%) & & \\
Operative Variables & $60.71 \pm 29.38$ & $63.16 \pm 30.19$ \\
Mean operative time, min &
\end{tabular}

BMI = Body Mass Index.
Table 2

Thirty-day postoperative complications in overall Achilles tendon rupture repair cohort.

\begin{tabular}{lll}
\hline Outcome & Count (Overall $\mathrm{N}=1164)$ & $\%$ of total \\
\hline Any complication & 23 & 2.0 \\
Superficial wound infection & 10 & 0.8 \\
Deep wound infection & 3 & 0.3 \\
Wound dehiscence & 3 & 0.3 \\
Pneumonia & 1 & 0.1 \\
Deep-vein thrombosis & 2 & 0.2 \\
Reoperation & 6 & 0.5 \\
\hline
\end{tabular}

Values listed as percentages.

Table 3

Multivariate Analysis of Risk Factors for Any Adverse Event.

\begin{tabular}{llc}
\hline Outcome & Odds ratio $(95 \% \mathrm{CI})$ & $p$ value \\
\hline Any adverse event & & \\
Obesity & $0.57(0.10-3.14)$ & 0.52 \\
History of Smoking & $2.66(0.71-10.03)$ & 0.15 \\
History of Diabetes & $1.21(0.51-14.26)$ & 0.25 \\
Age & $1.01(0.97-1.05)$ & 0.81 \\
Gender (Male) & $1.05(0.40-2.77)$ & 0.93 \\
History of Hypertension & $0.39(0.97-1.55)$ & 0.18 \\
History of COPD & $6.94(0.68-70.5)$ & 0.10 \\
\hline
\end{tabular}

( $2 \%$ vs. $2 \%, \mathrm{p}=0.981)$ or superficial SSI $(0.9 \%$ vs. $0 \%, \mathrm{p}=0.183)$. Operative time was significantly longer in HR group compared to healthy controls in the study $(63.16 \pm 30.2 \mathrm{~min}$ vs. $57.95 \pm 28.2 \mathrm{~min}$, $\mathrm{p}=0.002$ ). Logistic multivariate analysis did not reveal any patient characteristics included in the model to be significantly associated with the occurrence of an adverse event or superficial SSI (Table 3).

\section{Discussion}

The patient population in this study was relatively young and healthy with an average age of 44.8 and few comorbidities. This is consistent with the epidemiology of ATRs documented in other literature. $^{12-14}$ The controversy regarding optimal management of ATR largely centers on weighing the risk of surgical complications against potentially longer recovery time and suboptimal functional results if patients are managed nonoperatively. ${ }^{9,15}$ This study found a very low overall adverse event rate of $2 \%$ in the 30 -day postoperative period for primary surgical ATR repair.

The HR cohort of patients had a statistically significantly higher operative time relative to healthy controls without risk factors. However, this difference of six minutes between the risk-stratified groups is likely not clinically significant. Patients undergoing ATR repair with graft had significantly higher operative time than those patients undergoing ATR repair without graft but did not differ in adverse event rates.

Though in this study there was no increased risk of adverse events across comparisons, increased operative time has been implicated as a risk factor for postoperative infection in numerous studies. ${ }^{7,25} \mathrm{~A}$ comparison of operative and conservative management by Cukelji et al found a lower rate of complications in patients undergoing percutaneous ATR repair and noted a lower operative time in these patients. ${ }^{9}$ However, the sample size in their study was much smaller and specifically targeted athletes aged 25-40. Lower operative time did not correlate with lower incidence of complication in this study. However, it should be emphasized that the total recovery period for ATR extends far beyond the 30-day postoperative period.

Wound complications after ATR surgical repair have been documented as a concern that warrants consideration of nonoperative treatment for these injuries. ${ }^{8}$ A retrospective study of 60 operatively managed ATR patients by Marican et al found that $16.7 \%$ of patients 
suffered infectious complications. ${ }^{18}$ In contrast to this study, we did not find an association between obesity and risk of superficial wound infections. Infection control protocols and antibiotic prophylaxis are not documented in the ACS-NSQIP database, which makes it difficult to know if there were modifiable factors leading to lower infectious complications than rates previously reported in the literature.

As the population in the United States becomes more obese, concerns for increased risk of complications in patients with increased body habitus have risen. ${ }^{11}$ In this study, patients with a higher risk profile did not have higher rates of complications relative to healthy controls. This suggests that for patients undergoing ATR surgical repair with risk factors explored in this study such as obesity, diabetes, and smoking, there isn't a higher probability of surgical complications in the 30-day postoperative period compared to healthier patients. Surgical candidates for ATR repair with modifiable risk factors should nevertheless continue to be advised on the negative health effects of smoking, obesity, and diabetes. This is especially true given that complications typically associated with these conditions are likely to occur outside the 30-day postoperative period and are not captured in the NSQIP database. Age was not seen to impact 30-day complication rates, though previous studies have documented the potential benefits of ATR surgical repair vary based on patient age. ${ }^{5}$

The ACS-NSQIP database provides the advantage of spanning numerous hospitals and minimizes the caveat of single surgeon bias. The patient population and rates of complications documented may be more representative of the overall U. S. population than other studies that rely on outcomes from a small cluster of institutions. Though the patient population in this study was comparable in size to others in the literature, the ACS-NSQIP database contained relatively few ATR compared to other conditions it has been used to investigate. ${ }^{2,3,10}$ The small number of adverse events makes it difficult to draw broader conclusions based on statistical findings in this study as does the short follow up time. The logistic regression model only accounted for a small degree of the variation in adverse event occurrence. However, univariate analyses also did not find an association between patients in the HR cohort and adverse event occurrence.

There are also several limitations to this study inherent to the ACSNSQIP database. Patient outcomes are tracked up to thirty days after hospital admission and complications after this period are not documented. ${ }^{6}$ The 30-day postoperative period is not indicative of long-term outcomes, though it is useful for minimizing patient readmissions and allows for analysis of imminent risks after surgery. Given that rerupture may occur well outside the 30-day postoperative period and is a particular outcome of interest in ATR repair, it must be acknowledged that this limitation impedes the utility of this study's results. Pain, range of motion, and outcomes commonly used in orthopaedic surgery are also not documented in the ACS-NSQIP database. Furthermore, information on rehabilitation protocols, use of medical management such as thromboprophylaxis or antibiotic prohylaxis, and specified surgical technique would be beneficial for further analysis to better characterize predictors of a successful ATR repair. ${ }^{4}$ This is particularly relevant given results from a recent meta-analysis concluding recommendations for surgical treatment be made based on availability of functional rehabilitation. ${ }^{26}$

\section{Conclusions}

Repair of Achilles tendon ruptures has a very low rate of complications in the 30-day postoperative period even in patients with several complication rates. Patient characteristics were not seen to be predictive of adverse events in ATR operative cases in this study, though these results are limited in their scope. Providers can use results from this study to further inform patient-physician discussions weighing the risks and benefits of operative vs. non-operative treatment for primary ATRs in the 30-day postoperative period.

\section{Conflict of interest}

None.

None of the authors have financial or institutional disclosures to report related to the research in this paper.

\section{References}

1. Amendola A. Outcomes of open surgery versus nonoperative management of acute achilles tendon rupture. Clin J Sport Med. 2014;24(1):90-91. [Internet]. Available from:. http://www.ncbi.nlm.nih.gov/pubmed/2436601710.1097/JSM. 0000000000000064.

2. Basques BA, Webb ML, Bohl DD, Golinvaux NS, Grauer JN. Adverse events, length of stay, and readmission after surgery for tibial plateau fractures. J Orthop Trauma. 2015;29(March(3)):e121-6. [Internet], [cited 2015 Feb 26]. Available from:. http:// www.ncbi.nlm.nih.gov/pubmed/2516297510.1097/BOT.0000000000000231.

3. Bohl DD, Fu MC, Golinvaux NS, Basques BA, Gruskay JA, Grauer JN. The July effect in primary total hip and knee arthroplasty: analysis of 21,434 cases from the ACSNSQIP database. J Arthroplasty. 2014;29(July(7)):1332-1338. [Internet], [cited 2015 Feb 26]. Available from:. http://www.ncbi.nlm.nih.gov/pubmed/2463112510. 1016/j.arth.2014.02.008.

4. Braunstein M, Baumbach SF, Boecker W, Carmont MR, Polzer H, Early EM Development of an accelerated functional rehabilitation protocol following minimal invasive Achilles tendon repair deep vein thrombosis. Knee Surg Sports Traumatol Arthrosc. 2015(September (26))http://dx.doi.org/10.1007/s00167-015-3795-1. [Internet]. Available from:

5. Claessen FM, de Vos RJ, Reijman M, Meuffels DE. Predictors of primary achilles tendon ruptures. Sports Med. 2014;2014:1241-1259. http://dx.doi.org/10.1007/ s40279-014-0200-Z.

6. Cohen ME, Ko CY, Bilimoria KY, et al. Optimizing ACS NSQIP modeling for evaluation of surgical quality and risk: patient risk adjustment, procedure mix adjustment, shrinkage adjustment, and surgical focus. J Am Coll Surg. 2013;217(August (2)):336-346. [Internet], [cited 2015 Feb 6]. Available from:. http://www.ncbi.nlm. nih.gov/pubmed/2362822710.1016/j.jamcollsurg.2013.02.027.

7. Colman M, Wright A, Gruen G, Siska P, Pape HC, Tarkin I. Prolonged operative time increases infection rate in tibial plateau fractures. Injury. 2013;44(2):249-252. http://dx.doi.org/10.1016/j.injury.2012.10.032. [Internet]. Available from:.

8. Cooper MT. Acute Achilles tendon ruptures. Clin Sports Med. 2015;34(4):595-606. [Internet]. Available from:. http://linkinghub.elsevier.com/retrieve/pii/ S027859191500044710.1016/j.csm.2015.06.001.

9. Cukelj F, Bandalovic A, Knezevic J, Pavic A, Pivalica B, Bakota B. Treatment of ruptured Achilles tendon: operative or non-operative procedure? Injury. 2015 [Internet]. Available from:. http://linkinghub.elsevier.com/retrieve/pii/ S002013831500687710.1016/j.injury.2015.10.070.

10. Curran T, Zhang JQ, Lo RC, et al. Risk factors and indications for readmission after lower extremity amputation in the American College of Surgeons National Surgical Quality Improvement Program. J Vasc Surg. 2014;60(November(5)):1315-1324. [cited 2015 Feb 11], [Internet]. Available from:. http://www.ncbi.nlm.nih.gov/ pubmed/2498553610.1016/j.jvs.2014.05.050.

11. Guss D, Bhattacharyya T. Perioperative management of the obese orthopaedic patient. J Am Acad Orthop Surg. 2006;14(7):425-432 14/7/425 [pii]

12. Houshian S, Tscherning T, Riegels-Nielsen P. The epidemiology of achilles tendon rupture in a Danish county. Injury. 1998;29(9):651-654. http://dx.doi.org/10.1016/ S0020-1383(98)00147-8.

13. Huttunen TT, Kannus P, Rolf C, Felländer-Tsai L, Mattila VM. Acute achilles tendon ruptures: incidence of injury and surgery in Swedenbetween 2001 and 2012. Am J Sports Med. 2014;42(10):2419-2423. [Internet]. Available from:. http://www.ncbi. nlm.nih.gov/pubmed/2505698910.1177/0363546514540599.

14. Järvinen TA, Kannus P, Maffulli N, Khan KM. Achilles tendon disorders: etiology and epidemiology. Foot Ankle Clin. 2005;10(2):255-266. http://dx.doi.org/10.1016/j.fcl. 2005.01.013.

15. Jiang N, Wang B, Chen A, Dong F, Yu B. Operative versus nonoperative treatment for acute Achilles tendon rupture: a meta-analysis based on current evidence. Int Orthop. 2012;36(4):765-773. [Internet]. Available from. http://www.pubmedcentral.nih. gov/articlerender.fcgi? artid $=3311794 \&$ tool $=$ pmcentrez $\&$ rendertype $=$ abstract 10 1007/s00264-011-1431-3.

16. Józsa L, Kvist M, Bálint B, et al. The role of recreational sport activity in Achilles tendon rupture. A clinical, pathoanatomical, and sociological study of 292 cases. Am J Sport. Med. 1989;17(3):338-343. http://dx.doi.org/10.1177/ 036354658901700305.

17. Maffulli N, Waterston SW, Squair J, Reaper J, Douglas AS. Changing incidence of Achilles tendon rupture in Scotland: a 15-year study. Clin J Sport Med. 1999;9(3):157-160. [Internet]. Available from:. http://www.ncbi.nlm.nih.gov/ pubmed/1051234410.1097/00042752-199907000-00007.

18. Marican M, Fook-Chong S, Rikhraj I. Incidence of postoperative wound infections after open tendo Achilles repairs. Singapore Med J. 2015;56(10):549-554. [Internet]. Available from:. https://www.sma.org.sg/Publications/articles.aspx?ID = AFC4F2C5-ECD0-4509-A3EF-39E7B6EBF59210.11622/smedj.2015150.

19. Molina CS, Thakore RV, Blumer A, Obremskey WT, Sethi MK. Use of the national surgical quality improvement program in orthopaedic surgery. Clin Orthop Relat Res. 2014;473(5):1574-1581. [Internet]. Available from:. http://link.springer.com/10. 1007/s11999-014-3597-710.1007/s11999-014-3597-7.

20. Olsson N, Nilsson-Helander K, Karlsson J, et al. Major functional deficits persist 2 
years after acute Achilles tendon rupture. Knee Surg Sports Traumatol Arthrosc. 2011;19(8):1385-1393. http://dx.doi.org/10.1007/s00167-011-1511-3.

21. Scott A, Grewal N, Guy P. The seasonal variation of Achilles tendon ruptures in Vancouver, Canada: a retrospective study. BMJ Open. 2014;4(2):e004320. [Internet]. Available from:. https://www.ncbi.nlm.nih.gov/pubmed/?term $=$ The + seasonal

+ variation + of + Achilles + tendon + ruptures + in + Vancouver $\% 2 \mathrm{C}+$ Canada $\% 3 \mathrm{~A}+\mathrm{a}+$ retrospective + study.

22. Soroceanu A, Sidhwa F, Aarabi S, Kaufman A, Glazebrook M. Surgical versus nonsurgical treatment of acute Achilles tendon rupture: a meta-analysis of randomized trials. J Bone Joint Surg Am. 2012;94(23):2136-2143. [Internet]. Available from:. http://www.pubmedcentral.nih.gov/articlerender.fcgi?artid = 3509775\&tool = pmcentrez\&rendertype $=$ abstract10.2106/JBJS.K.00917.

23. Suchak AA, Bostick G, Reid D, Blitz S, Jomha N. The incidence of Achilles tendon ruptures in Edmonton, Canada. Foot Ankle Int. 2005;26(11):932-936. http://dx.doi. org/10.1177/107110070502601106.

24. Wilkins R, Bisson LJ. Operative versus nonoperative management of acute Achilles tendon ruptures: a quantitative systematic review of randomized controlled trials. Am J Sports Med. 2012;40(9):2154-2160. [Internet]. Available from:. http://www. ncbi.nlm.nih.gov/pubmed/2280227110.1177/0363546512453293.

25. Zalavras CG, Christensen T, Rigopoulos N, Holtom P, Patzakis MJ. Infection following operative treatment of ankle fractures. Clin Orthop Relat Res. 2009;467(7):1715-1720. http://dx.doi.org/10.1007/s11999-009-0743-8.

26. Zhang $\mathrm{H}$, Tang $\mathrm{H}$, He $\mathrm{Q}$, et al. Surgical versus conservative intervention for acute achilles tendon rupture. Medicine (Baltimore). 2015;94(45):e1951. [Internet].

Available from:. http://content.wkhealth.com/linkback/openurl?sid = WKPTLP:landingpage\&an =00005792-201511110-0002210.1097/MD. 0000000000001951. 